\title{
Phonon-Induced Electrical Conductance in Semiconductors
}

\author{
K. LaBmann and W. Burger \\ Universität Stuttgart, Physikalisches Institut, Teil 1, \\ Pfaffenwaldring 57, D-7000 Stuttgart 80, Fed. Rep. of Germany
}

The bath of thermal phonons is a well-known source of impedance to electronic conduction in solids. Turning the tables, a change in electronic conductance may be used for phonon detection, as will be discussed in the following.

Since the phase veloctty $\omega / k$ in most cases 18 much smaller for phonons than for electrons in the interaction event the transfer of energy $n \omega$ is negligible for the electron whereas the change of momentum rik may be substantial. Thus, a phonon current will help to direct electron motion. This so-called phonon drag effect shows up in the large thermoelectric power of semiconductors at low temperatures. Activation of electrons by energy transfer in phonon scattering is important in doped semiconductors at low temperatures in situations where the electrons are immobilised in shallow potential wells and is evident in the exponential temperature dependence of conductance due to carrier freeze-out and hopping conductivity. Both aspects of electron-phonon interaction in semiconductors, drag and activation, have been applied for phonon detection by phonon-induced conductance.

The former possibility has been utilized as so-called transmitted phonon drag in $G e$ and $S 1$ by $K$. HÜBNER and others (/1/ and references therein) in a series of experiments at liquid nitrogen and liquid helium temperatures. A typlcal set-up is sketched in Fig. 1: A slab of the crystal to be investigated is n-doped on two opposite faces. An electric field $E_{1}$ applied to the top n-layer causes an electron current which imparts some of its resultant momentum to the phonon distribution. Those of these perturbed phonons which propagate through the middle layer and penetrate into the bottom n-layer drag

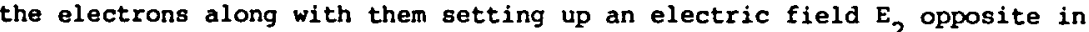
phase to the input field $E_{1}$. The variation of the ratio $E_{2} / E_{1}^{2}$ with experimental parameters such as thickness or doping of the middle layer, surface roughness, and bath temperature is a measure of the relative transmitted momentum. Though interesting and plausible dependencies on these parameters have been observed, the detailed interpretation suffered from insufficient information on the relevant phonon spectrum involved.

A first example for phonon activation is the technique of thermally stimulated currents $/ 2 /$ where nonequilibrium distributed electrons are

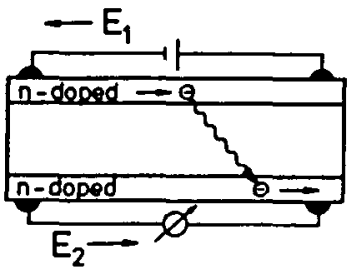

Fig. 1 Scheme of phonon generation and detection in the case of transmitted phorion drag in $\mathrm{Si}$ and $\mathrm{Ge}$. Thickness of doped layers several $\mu \mathrm{m}$, thickness of middle layer ranging from $50 \mathrm{~mm}$ to several $\mathrm{mm}$. /1/ 
liberated from deep traps by thermal phonons during a slow temperature sweep allowing spectral information with resolution of the order of $k T$. Such an equilibrium phonon technique does not distinguish between one- or multiphonon processes and the trap depth may be larger than the Debye frequency. (DLTS /3/ may be regarded as a variant of this method.)

Another possibllity of phonon-induced conductance is associated with the complementary process where nonequilibrium phonons excite electrons from their equilibrium positions. Three types of phonon-activated current may be envisaged in homogeneous semiconductors: hopping conduction in compensated or highly doped material, carrier liberation from shallow traps, and current due to band-band transitions in narrow bandgap semiconductors.

Phonon spectroscopy might give valuable information in all three cases: the distribution of the hopping states in energy and distance, the Coulomb gap and the Hubbard gap /4/ may be directly accessible, the elementary processes in carrier recombination to shallow traps /5/ may be investigated by the reverse process of activating bound electrons to conducting states and finally the electron-phonon interaction in narrow bandgap semiconductors $/ 6 /$ may be studied by this type of experiment.

As regards activated conductance due to nonequilibrium phonons, the first experiments have been by ASCARELLI /7/ and ZYLBERSZTEJN /8/ where phonons generated by hot electrons in Ge were detected by a conductivity change in a damaged surface layer biassed below avalanche threshold. However, details of the detection process were not investigated.

A first investigation on the possibility of ionisation from shallow traps has been made by CRANDALL $/ 9 /$ for shallow donors in GaAs $\left(E_{B}-6\right.$ meV). The set-up used was quite analogous to Fig. 1 with $500 \mathrm{\mu m}$ thick ${ }^{B}$ semiinsulating material covered by $40 \mu \mathrm{m}$ thick, moderately n-doped, top and bottom layers. One of the layers was supposed to generate high-frequency phonons from the recombination of electrons liberated by the avalanche process and the other layer was thought to detect these phonons by the reverse process of ionizing the neutral donors. However, no direct measurement of the phonon energies involved was possible. The same applies to the somewhat modified phonothermal detection scheme (i.e. excitation by a nonequilibrium phonon from ground state to an excited state and from there by phonons of the thermal bath to a currentcarrying state) as proposed by ULBRICH $/ 10 /$ in an experiment with optically generated phonons in GaAs.

WIGMORE /11/ used GaAs doped with acceptors $\mathrm{Zn}$ or $\mathrm{Cd}$ ( $\mathrm{E}_{\mathrm{B}}$ about $30 \mathrm{meV}$ ) for bolometric detection of heat pulses and microwave ultrasonic pulses. From the temperature dependence of the electrical conductivity $/ 12 /$ it was concluded that hopping activation was the process responsible for the phonon detection.

Since superconducting tunnel junctions are phonon generators with well established spectral properties $/ 13 /$ we have used them to analyze the highfrequency phonon detection by an epitaxial layer of $\mathrm{n}$-GaAs (Sn $5 \times 10^{15} \mathrm{~cm}^{-3}$. compensation about 58 ) on a $3 \mathrm{~mm}$ thick semiinsulating substrate. From measurement of the temperature dependence of the conductance we $f$ ind activated behaviour between $6 \mathrm{~K}$ and $11 \mathrm{~K}$ with $\mathrm{E}=5.8 \mathrm{meV}$ corresponding to the donor binding energy and a variation as $\exp \left(-T_{0} / T\right)^{1 / 4}$ below $2.5 \mathrm{~K}$ characteristic for variable range hopping with $T_{0}=0.072 K$, so, both types of phonon activated current might be expected in a phonon spectroscopy experiment. Fig. 2 shows the results obtained with a $S n$ junction generator. Curve $A$ is the 


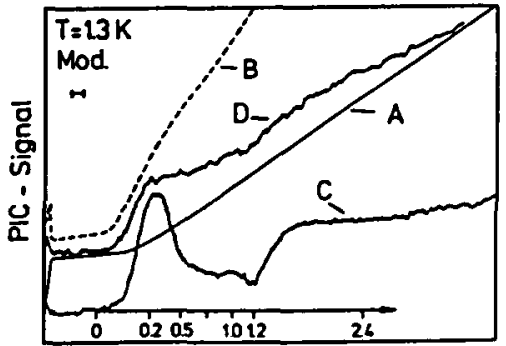

Phonon energy $E_{\text {ph }}[\mathrm{meV}]$

Fig. 2 PIC-signal of an epitaxial layer of $n$-GaAs (thickness $60 \mu \mathrm{m}$.

Sn concentr. $5 \times 10^{15} \mathrm{~cm}^{-3}$, compensation 0.05 )

with $\mathrm{Sn}$ junction as phonon generator. A: $\uparrow$ junction bias $\mathrm{U}$,

$\rightarrow$ junction current $I$,

$I=155 \mathrm{~mA}$ at $U=4 \Delta / \mathrm{e}=2.36 \mathrm{meV}$

B: Calculated for constant

energy-detection sensitivity

C: PIC-signal, helium contact

D: PIC-signal, oil coverage

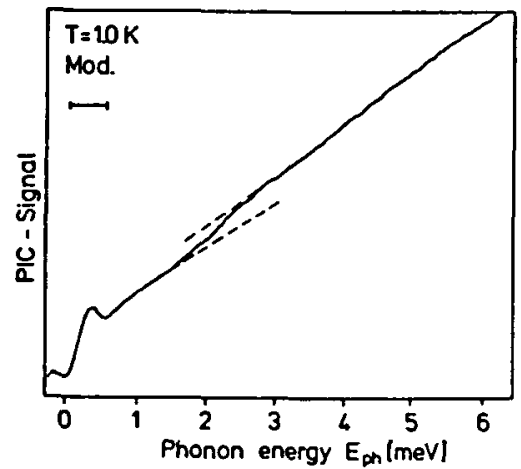

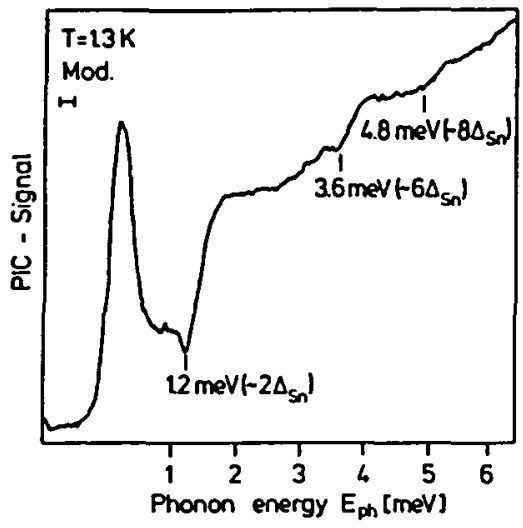

Fig. 3 PIC-signal corresponding to condition of $\mathrm{C}$ in Fig. 2,but measured to higher junction bias.

At multiples of the gap

down-conversion of high-energy

phonons modulo $2 \Delta$ by pair breaking increases the number of low-frequency phonons and thus PIC because of the low-frequency sensitivity of hopping. Only few phonons above the gap are emitted from the Sn film.
Fig. 4 PIC-signal corresponding to Fig. 3 but with Al junction as phonon generator instead of $\mathrm{Sn}$. The change in slope at $0.2 \mathrm{meV}$ and the decay at $0.35 \mathrm{meV}$ are the same as for $\mathrm{Sn}$. Despite the high-frequency phonons emitted by the Al junction, no threshold is found. The changes in slope at $1.5 \mathrm{meV}$ and $2.8 \mathrm{meV}$ and some small wiggles are reproducible for different Al junctions.

dc-characteristic of the junction, the energy scale being that of the maximum primary relaxation phonons for generator bias beyond the gap voltage. Curve $B$ would be expected qualitatively for energy detection. Curve $C$ shows the phonon -induced current (PIC) signal: After the expected rise beyond the gap voltage there is a change in slope near $0.2 \mathrm{meV}$ followed by a decay starting at $0.35 \mathrm{mev}$. This latter decay is due to the treshold for increased phonon transmission into liquid helium in contact with the generator junction /14/. This contact can be spoiled by a coverage of the junction with oil which therma- 
lizes the phonons transmitted to it. The effect is shown in curve $D$ where the Kapitza threshold has disappeared, whereas the reduced detector sensitivity starting near $0.2 \mathrm{meV}$ clearly remains. This reduction for high-frequency phonons is manifest also in reduced signal of $A$ in the region of gap-bias where $1.2 \mathrm{meV}$ phonons are generated. After partial down-conversion of these phonons by the oil there is a relative increase of PIC at this bias. Fig. 3 shows the PIC with a $\mathrm{Sn}$ generator measured to nominally higher energies. Since reabsorption in $\mathrm{Sn}$ prevents sizeable emission of phonons above $2 \Delta \mathrm{Sn}=1.18 \mathrm{meV}$,

the observed structure is astonishing at first glance. Most of it can, however, be understood by this very reabsorption (which leads to a down-conversion of the high-frequency relaxation phonons modulo $2 \Delta \mathrm{Sn}^{\prime}$ taking into account the Kapitza threshold and the detector preference for low-frequency phonons as is discussed in detail in $/ 15 /$.

Thin Al generators do not have this reabsorption limit /13/. Fig. 4 shows the corresponding PIC result. Apart from the low-frequency reduction just discussed for Fig. 2 we find distinct slope changes near $1.5 \mathrm{meV}$ and $2.8 \mathrm{meV}$ together with small structures at several frequencles reproducible for different generator junctions. No clear-cut correlation of these structures to any energy differences starting from the ground state of the isolated donor could be made. The overall frequency dependence appears to be rather smooth. It must be kept in mind, however, that part of the nominally high-frequency signal may be made up by low-frequency decay products from phonon scattering in the substrate, in the epitaxial layer, in the junction, and at the interfaces. The details of this have to be sorted out in further experiments. What can be said so far is that the signal is mainly due to hopping activation. whereas ionization of the donors plays no significant role. The reason for this may be either the absorption of the corresponding high-frequency phonons in the substrate or the intrinsic difficulty to excite electrons from the ground state by phonons, because the extended bound state cannot take up the large phonon $k$-vector connected with the energy difference.

Ionization by phonons has been demonstrated by us $/ 16 / / 17 /$ for a different class of shallow traps, namely $D^{-}$and $A^{+}$centers in $S i$ and Ge first found by FIR-measurements $/ 18$. In complete analogy to atomic hydrogen, neutral effective-mass donors and acceptors can trap a second electron or hole respectively with binding energies of the order of mev (about $1 / 20$ of the neutral impurity binding energy, whereas the extent of the wavefunction is larger by only about a factor of 2 which makes the relation to the involved phonon wavelength more favourable for one-phonon transitions). As an example the variation of the PIC threshold with boron concentration is shown in Fig.5. The high resolution and sensitivity of the method is evident from the curve for the smallest concentration of $5 \times 10^{12} \mathrm{~cm}^{-3}$ of boron. The free carriers to be trapped by the neutral impurities were produced by illumination with visible light or, alternatively with room temperature radiation. It is remarkable that already for $5 \times 10^{13} \mathrm{~cm}^{-3}$ a broadening of the rise is observed corresponding to a distance of 125 Bohr radii of the neutral boron. Such a broadening and finally a shifting at higher concentrations is attributed to the interaction of two or more neutral impurities in the capture and binding of the extra carrier. The interaction over large distances observed here for the first time may be a consequence of the slowly decaying fong-range part of $\mathrm{H}$-wave function. Similar results have been obtained for $\mathrm{Al}$ and $\mathrm{Ga}^{\dagger}$ (somewhat smaller binding energy than for $B^{+}$) and $I^{+}$(Binding energy about 6 meV corresponding to the larger depth of this acceptor).

A phonon energy of $12 \mathrm{meV}$ is quite close to the limiting frequency for some of the TA-branches in $\mathrm{Si}$. So, isotope scattering and anharmonic decay become 


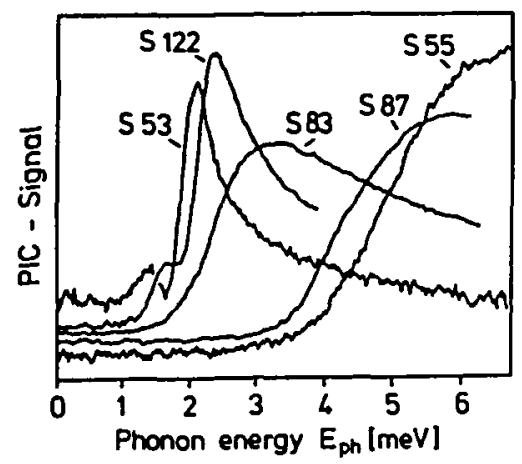

Fig. 5 PIC threshold of $\mathrm{B}^{+}$in Si: Dependence on boron concentration.

S53: $5.0 \times 10_{13}^{12} \mathrm{~cm}_{-3}^{-3}$

S122: $5.0 \times 10_{14}^{13} \mathrm{~cm}_{-3}^{-3}$

S83: $9.5 \times 10_{15}^{14} \mathrm{~cm}_{-3}^{-3}$

S87: $5.4 \times 10^{15} \mathrm{~cm}^{-3}$

The precursor resolved for the steeper thresholds of $\$ 53$ and $\$ 122$ is a feature of the spectrum emitted by the Al junction.

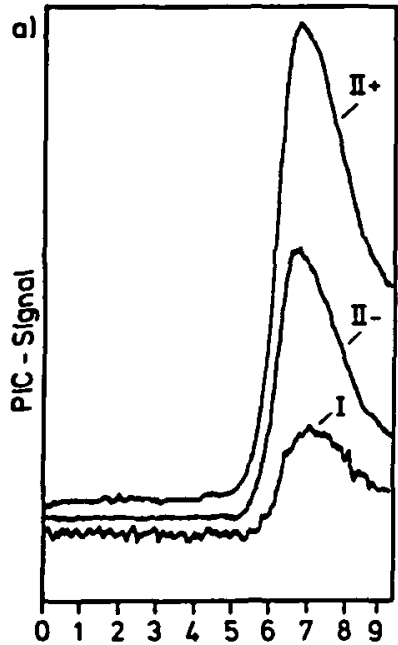

b)
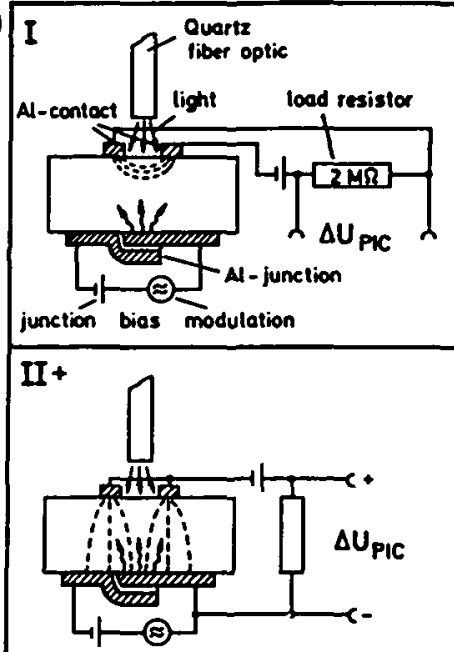

Phonon energy $E_{p h}[m e V]$

Fig. 6 a) Variation of PIC-signal form and strength for different probing distances from generator junction obtained by different measuring configurations in the case of In $^{+}$in $\mathrm{Si}$.

b) Configuration I: Probing zone opposite to junction ( $-2.5 \mathrm{~mm})$ Configuration IIt: Probing zone near junction since holes produced by illumination are driven there by the bias Configuration II-: Intermediate

increasingly important. Measuring with different electrode configurations as Indicated in Fig. $6 \mathrm{~b}$ we get a qualitative measure of the phonon mean free path: configuration I probes the region opposite to the phonon generator, whereas II measures the conductivity change along the phonon path. In this case an additional distinction is possible by the polarity of the bias: IIt as indicated in Fig. $6 \mathrm{~b}$ dfaws the holes generated by the illumination to the junction so that the $\mathrm{A}^{+}$centers produced in this region can probe phonons with short mean free paths. For the reverse bias, the probing region is shifted away from the junction. The effect on the measuring signal is shown in Fig. 6 a for the case of $\mathrm{In}^{+}$: for the largest probing distance we have the smallest 
signal. Also the decay beyond the threshold is steeper for the longer phonon path which would be expected from the frequency dependence of phonon

scattering. So from this type of measurement an estimate of the phonon mean free path and its frequency dependence can be obtained. An example for this is given in $/ 18 /$.

An important result of these experiments is the reproducible demonstration of the feasibility of phonon spectroscopy with thin Al generators up to at least $12 \mathrm{meV}(/ 17 / / 19 /)$. By this it is ascertained that the negative phonoIonization result in the case of neutral donors in GaAs is not due to the limitations of the Al generator.

Another point to be made is the following: The first excited state(s) above the ground state of the neutral impurity should have a long lifetime if excited at low temperatures because of the large phonon wavevector associated with recombination by single phonon emission. If a long-lived excited state of the neutral impurity were produced by the illumination we might have expected additional thresholds at about $11 \mathrm{meV}$ or below e.g. for Si (P). So far, we could not observe corresponding signals either in silicon or in germanium.

As mentioned above, relaxation phonons with energies larger than the gap are reabsorbed and down-converted within a Sn junction generator because the films cannot easily be made thin enough. Nevertheless, the small amount of beyond-gap phonons emitted is readily detected by a conductivity change at the $P$ threshold $\left(E_{p_{-}}-2 \mathrm{mev}\right)$ as shown by Curve $A$ in Fig. 7. Curve B shows the PIC-signal for an Al junction generator for comparison. Since these highfrequency phonons from a Sn generator could be detected only with difficulty by other methods this result demonstrates the sensitivity for high-frequency phonon analysis. The possibility to shift the threshold by uniaxial pressure might be an additional advantage for phonon spectroscopy.

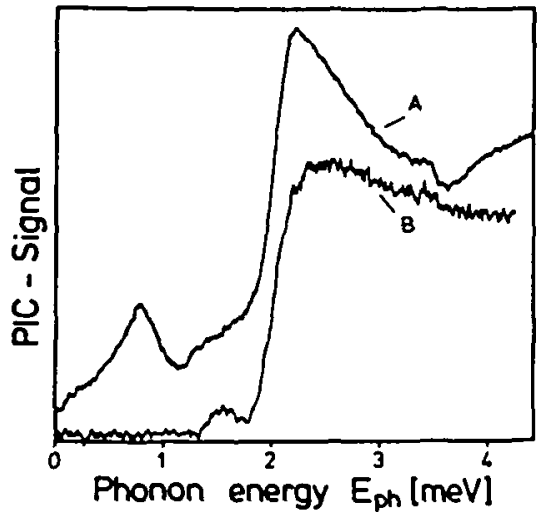

Fig. 7 High-frequency phonon emission from a $\mathrm{sn}$ junction as detected by PIC of Si(P) (Curve A) Junction thickness $150 \mathrm{~nm}$,

(P) $=6 \times 10^{14} \mathrm{~cm}^{-3}$

Curve $B$ is obtained with an

Al junction generator.

In both cases preceding the threshold at 2 meV a precursor is found at a distance of the corresponding gap which is due to relaxation of excited quasiparticles having tunnelled through the barrier.

For better time and space resolved measurements one needs small and low ohmic structures analogous to the epitaxial cas layer with finger electrodes evaporated on it (ISHIGURO et al. /20/), used as a detector for heat pulses. The details of the detection mechanism in this case have not been investigated so far. The frequency dependence of the detection sensitivity would be interesting to measure by phonon spectroscopy. as described above for the GaAs epitaxial layex.

It is seen from these examples that the combination of tunnel junction phonon generators with the detection by conductivity changes from activated 
carriers is a powerful experimental tool because of the well-defined and easily controllable spectral properties of the former and the high sensitivity with spectral resolution at high frequencies of the latter. It opens new and direct possibilities to investigate phonon participation in electronic processes in semiconductors at low temperatures in an interesting range of frequencies.

E. Bauser of MPIF in Stuttgart kindly provided us with the GaAs samples. We are grateful to $W$. Zulehner of Hacker Chemitronic for many vell characterized silicon samples.

This work is supported by the Deutsche Forschungsgemeinschaft.

\section{References}

1. R. Gereth and K. Hübner: Solid-St. Electron. 10, 935 (1967)

2. R.H. Bube in: Photoelectronic Materials and Devices, ed. by

S. Larach, (Van Nostrand, Inc.. New York, 1965) p.113

3. D.V. Lang: J. Appl. Phys. 45, 3023 (1974)

4. B.I. Shklovskii and A.I. Efros: Electronic Properties of Doped Semiconductors, Springer Series in Sol. State Science 45 (Springer, Berlin, Heidelberg 1985)

5. F. Beleznay and L. Andor: Solid-St. Electron. 21, 1305 (1978)

6. W. Zawadzki and P. Boguslawski: Phys. Rev. Lett. 31, 1403 (1973)

7. G. Ascarelli : Phys. Rev. Lett. 5, 367 (1960)

8. A. Zylbersztejn : Phys. Rev, Lett. 19. 838 (1967)

9. R.S. Crandall : Solid-St. Commun. 7, 1109 (1969)

10. R. Ulbrich: In Monequilibrium Phonon Dynamics, ed. by W.E. Bron, NATO ASI Series B124, 101 (1985)

11. J.K. Wigmore: J. Appl. Phys. 41, 1996 (1970)

12. J.K. Wigmore and P. Tihabologang: Appl. Phys.Lett. 42, 685 (1983)

13. W. Eisenmenger: In Nonequilibrium Superconductivity, Phonons, and Kapitza Boundaries, ed. by E. Gray, NATO ASI Series B65, 73 (1981)

14. O. Koblinger, U. Heim, M. Welte, and $H$. Eisenmenger: Phys. Rev. Lett. 51, 284 (1983)

15. W. Burger: Thesis, Stuttgart 1986

16. W. Burger and K. Laßmann: Phys. Rev. Lett. 53, 2035 (1984)

17. W. Burger and K. LaBmann: Phys. Rev. B33, 5868 (1986)

18. S. Narita: J. Phys. Soc. Jpn. 49 Suppl. A, 173 (1980)

19.. . Burger and $K$. Laßmann: These Proceedings

20. T. Ishiguro and S. Morita: Appl. Phys. Lett. 25, 533 (1974) 